\title{
Exact Free Energy Functional for a Driven Diffusive Open Stationary Nonequilibrium System
}

\author{
B. Derrida \\ Laboratoire de Physique Statistique, 24 rue Lhomond, 75231 Paris Cedex 05, France \\ J. L. Lebowitz用 and E. R. Speerf \\ Department of Mathematics, Rutgers University, New Brunswick, NJ 08903
}

\begin{abstract}
We obtain the exact probability $\exp [-L \mathcal{F}(\{\rho(x)\})]$ of finding a macroscopic density profile $\rho(x)$ in the stationary nonequilibrium state of an open driven diffusive system, when the size of the system $L \rightarrow \infty$. $\mathcal{F}$, which plays the role of a nonequilibrium free energy, has a very different structure from that found in the purely diffusive case. As there, $\mathcal{F}$ is nonlocal, but the shocks and dynamic phase transitions of the driven system are reflected in non-convexity of $\mathcal{F}$, in discontinuities in its second derivatives, and in non-Gaussian fluctuations in the steady state.
\end{abstract}

PACS numbers: 05.70.Ln, 05.40.-a, 82.20.-w

The behavior of macroscopic systems which carry steady currents is one of the central problems in nonequilibrium statistical mechanics [1]. Of particular interest are stationary nonequilibrium states (SNS) maintained by contact with infinite reservoirs at the system boundaries and subjected to bulk driving forces. A paradigm of such systems is a fluid in contact with a thermal reservoir at temperature $T_{a}$ at the top and $T_{b}>T_{a}$ at the bottom, for which gravity supplies the bulk force (the RayleighBénard system [2]); the system exhibits dynamic phase transitions corresponding to the formation of different patterns of heat and mass flow as the parameters are varied. By contrast, if $T_{a}>T_{b}$ the system has no instabilities. These dynamic transitions are not understood, at present, in terms of a microscopically derived free energy, despite various promising attempts [3]. Here we obtain the analogue of such a free energy for the SNS of a model system which, despite its simplicity, has some dynamic transitions.

We consider the one-dimensional asymmetric simple exclusion process (ASEP) on a lattice of $L$ sites [4, 5. Each site $i, i=1, \ldots, L$, is either occupied by a single particle $\left(\tau_{i}=1\right)$ or is empty $\left(\tau_{i}=0\right)$. In the interior of the system $(2 \leq i \leq L-1)$, a particle attempts to jump to its right neighboring site with rate 1 and to its left neighboring site with rate $q$ (with $0 \leq q<1$ ), succeeding if the target site is empty. At the boundary site $i=1$ $(i=L)$ particles jump only to the right (left). These boundary sites are also connected to particle reservoirs: if site 1 is empty, it becomes occupied at rate $\alpha$ (by a particle from the left reservoir); similarly, if site $L$ is occupied, the particle may jump into the right reservoir at rate $\beta$.

This dynamics produces an SNS for which we calculate the large $L$ behavior of $P_{L}(\{\rho(x\}) \simeq \exp [-L \mathcal{F}(\{\rho(x)\})]$, the probability for observing microscopic configurations corresponding, in the limit $L \rightarrow \infty, x=i / L$, to the macroscopic density profile $\rho(x), 0 \leq x \leq 1$.
$\mathcal{F}(\{\rho(x)\})$ is generally called the large deviation functional (LDF) in the mathematical literature [6]; one always has $\mathcal{F}(\{\rho(x)\}) \geq 0$, with equality holding only if $\rho(x)=\bar{\rho}(x)$, a typical density profile in the system, so that atypical profiles are observed with exponentially small probability for large $L$. For equilibrium systems $\mathcal{F}(\{\rho(x)\})$ is given as the difference in free energies for $\rho(x)$ and for $\bar{\rho}(x)$.

In our previous work [7] we considered the case $q=1$, in which the bulk dynamics are symmetric. We obtained there an exact $\mathcal{F}_{s}(\{\rho\})$ which, unlike the free energy in equilibrium, was nonlocal, reflecting the generic presence of long range correlations in SNS [8]. Due to the purely diffusive nature of the bulk dynamics, $\mathcal{F}_{s}$ did not exhibit any phase transitions or instabilities. This is quite different from the asymmetric case considered here which has, due to the driven nature of the bulk dynamics, not only long range correlations but also a rich phase diagram including phase transitions and shocks $[$, 5 , 9. It is thus closer to a real fluid and gives rise to a correspondingly more complex $\mathcal{F}(\{\rho\})$.

Before describing our new results about $\mathcal{F}$ we summarize some known properties of the open ASEP [10]. We restrict ourselves in this paper to $\alpha=(1-q) \rho_{a}$ and $\beta=(1-q)\left(1-\rho_{b}\right)$ with $0 \leq \rho_{a}, \rho_{b} \leq 1$; the parameters $\rho_{a}$ and $\rho_{b}$ represent the densities in the left and right reservoirs. For $\rho_{a}=\rho_{b}=r$ the stationary measure is just a product measure [5, 10] with uniform density $r$. This means that all static (i.e. single time) properties of the system, including $\mathcal{F}$, are the same as for an equilibrium lattice gas with fugacity $z=r /(1-r)$. For this system the LDF is given by [6, []

$$
\begin{aligned}
\mathcal{F}_{e q} & (\{\rho(x)\}) \\
= & \int_{0}^{1}\left[\rho(x) \log \frac{\rho(x)}{r}+(1-\rho(x)) \log \frac{1-\rho(x)}{1-r}\right] d x .
\end{aligned}
$$

Now recall that if an infinite system with ASEP dynamics is started in an initial state with a macroscopic 


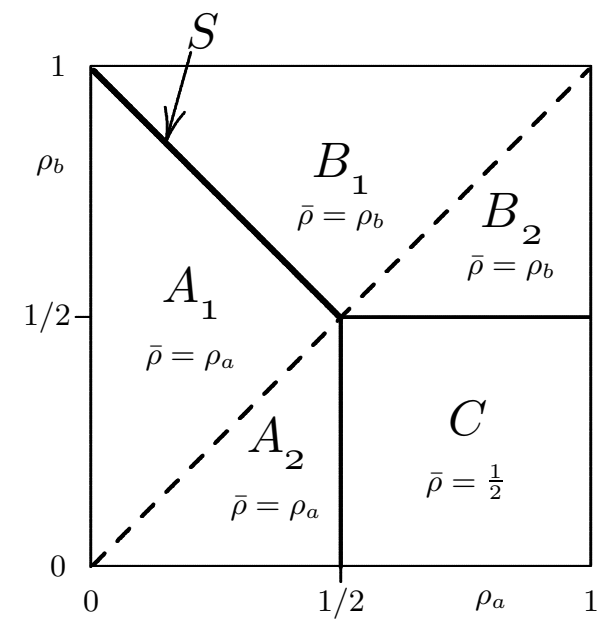

FIG. 1: The phase diagram of the open ASEP

density profile $\rho(x, 0)=\rho_{a}$ for $x<X, \rho(x, 0)=\rho_{b}$ for $x>X$, then when $\rho_{a}<\rho_{b}$ the time evolved $\rho(x, t)$ will maintain a sharp shock which will move with velocity $V=(1-q)\left(1-\rho_{a}-\rho_{b}\right)$, while when $\rho_{a}>\rho_{b}, \rho(x, t)$ will smooth out as in a "fan": $\rho(x, t)=\rho_{a}$ if $x \leq x_{a}(t)$, $\rho(x, t)=\rho_{a}+\left(\rho_{b}-\rho_{a}\right)\left(x-x_{a}(t)\right) /\left(x_{b}(t)-x_{a}(t)\right)$ if $x_{a}(t)<x<x_{b}(t)$, and $\rho(x, t)=\rho_{b}$ if $x_{b}(t) \leq x$, with $x_{\alpha}(t)=X+(1-q)\left(1-2 \rho_{\alpha}\right) t, \alpha=a, b$. In each case (unless $V=0$ ) the system will attain, as $t \rightarrow \infty$, a constant density $\bar{\rho}$ in any finite region. This gives an understanding [9] of the phase diagram shown in Fig. 1 for the system with open boundaries. For $\rho_{a}<\rho_{b}$, the shock will move to the right boundary when $V>0$, leaving behind the constant density $\bar{\rho}=\rho_{a}$ (phase $A_{1}$ ), and to the left boundary when $V<0$, yielding $\bar{\rho}=\rho_{b}$ (phase $\left.B_{1}\right)$. On the line $S(V=0)$ a typical $\bar{\rho}(x)$ is no longer constant, but corresponds to a shock at some point $s$, uniformly distributed on $[0,1]$, where $\bar{\rho}(x)$ jumps from $\rho_{a}$ to $\rho_{b}$, i.e. $\bar{\rho}(x)=\rho_{s}(x) \equiv \rho_{a} \Theta(s-x)+\rho_{b} \Theta(x-s)$ with $\Theta$ the Heaviside function [10]. This line corresponds to a first order phase transition, with $\bar{\rho}$ discontinuous across $S$. For $\rho_{a}>\rho_{b}$ (phases $A_{2}, B_{2}$, and $C$ ) one sees the constant density, $\rho_{a}, \rho_{b}$, or $1 / 2$, which would have resulted from the fanlike behavior in the infinite system.

We now turn to our new results. The line $\rho_{a}=\rho_{b}$, which separates what we will call the shock region $\rho_{a}<$ $\rho_{b}$ from the fan region $\rho_{a}>\rho_{b}$, is irrelevant for $\bar{\rho}$ but plays a crucial role in the LDF. Defining

$$
\begin{gathered}
g(h, f)=h \log [h(1-f)]+(1-h) \log [(1-h) f], \\
K\left(\rho_{a}, \rho_{b}\right)=\log \bar{\rho}(1-\bar{\rho}),
\end{gathered}
$$

with $\bar{\rho}$ given in Fig. 1, we find: for $\rho_{a}>\rho_{b}$,

$$
\begin{aligned}
\mathcal{F}\left(\{\rho(x)\} ; \rho_{a}, \rho_{b}\right) & \\
& =-K\left(\rho_{a}, \rho_{b}\right)+\sup _{F(x)} \int_{0}^{1} d x g(\rho(x), F(x)),
\end{aligned}
$$

where the sup is over all monotone nonincreasing functions $F(x)$ which satisfy $\rho_{a} \geq F(x) \geq \rho_{b}, 0 \leq x \leq 1$; for $\rho_{a}<\rho_{b}$,

$$
\begin{aligned}
& \left.\mathcal{F}\left(\{\rho(x)\} ; \rho_{a}, \rho_{b}\right)\right)=-K\left(\rho_{a}, \rho_{b}\right) \\
& +\inf _{0 \leq y \leq 1}\left\{\int_{0}^{y} d x g\left(\rho(x), \rho_{a}\right)+\int_{y}^{1} d x g\left(\rho(x), \rho_{b}\right)\right\}
\end{aligned}
$$

The fact that the function $F(x)$ in (1) is required to be monotone makes it, like $y$ in (5), depend on the global form of $\rho(x)$, so $\mathcal{F}$ is a nonlocal functional of $\rho(x)$. It can be shown [11] that the optimal $F$ in (14), which we denote by $F_{\rho}$, is constructed as follows: let $G_{\rho}$ be defined as the concave envelope of the function $\int_{0}^{x}(1-\rho(y)) d y$; then $G_{\rho}^{\prime}(x)$ is monotone,

$$
F_{\rho}(x)=G_{\rho}^{\prime}(x) \quad \text { if } \quad \rho_{b} \leq G_{\rho}^{\prime}(x) \leq \rho_{a},
$$

and $F_{\rho}(x)=\rho_{a}\left(F_{\rho}(x)=\rho_{b}\right)$ where $G_{\rho}^{\prime}(x) \geq \rho_{a}$ $\left(G_{\rho}^{\prime}(x) \leq \rho_{b}\right)$. Note that $F_{\rho}(x)$ need not be continuous; it will generally consist of strictly decreasing pieces, where $F_{\rho}(x)=1-\rho(x)$, flat pieces, where $F_{\rho}(x)=\rho_{a}$, $F_{\rho}(x)=\rho_{b}$, or $F_{\rho}(x)$ is obtained from $1-\rho(x)$ by a Maxwell construction (the integrals of $F_{\rho}(x)$ and of $1-\rho(x)$ over the latter intervals being equal), and possible jumps downward.

Before going on to discuss the derivation of (田) and (5) we describe some consequences.

(a) It can be verified that $\mathcal{F}\left(\{\rho(x)\} ; \rho_{a}, \rho_{b}\right) \geq 0$; equality occurs only when $\rho(x)=\bar{\rho}$ as given in the phase diagram, except on the first order line $S$, where it is the shock (typical) configurations $\rho_{s}(x)$ which satisfy $\mathcal{F}\left(\left\{\rho_{s}(x)\right\} ; \rho_{a}, \rho_{b}\right)=0$ for all $s \in[0,1]$.

(b) $\mathcal{F}\left(\{\rho(x)\} ; \rho_{a}, \rho_{b}\right)$ given by (4i) is a convex functional of $\rho(x)$ in the fan region $\rho_{a} \geq \rho_{b}$, since it is the supremum over $F$ of $\int_{0}^{1} g(\rho, F) d x$ with $g$ a locally convex function of $\rho$ for every $F$. This is similar to what happens in the symmetric case [7]. In the shock region $\rho_{a}<\rho_{b}$, on the contrary, this is not true; for every $\rho_{a}, \rho_{b}$ there are profiles $\rho(x)$ near which $\mathcal{F}$ is not convex. This is easily verified on the line $S$ where a superposition $\rho(x)=$ $\lambda \rho_{s}(x)+(1-\lambda) \rho_{t}(x), s \neq t$, satisfies $\mathcal{F}(\{\rho(x)\})>0$ for $0<\lambda<1$.

(c) The LDF in the fan region $\rho_{a}>\rho_{b}$ has similarities besides convexity to that in the symmetric case. In particular it is easy to see from (41) that $\mathcal{F}(\{\rho(x)\}) \geq$ $\mathcal{F}_{e q}(\{\rho(x)\})$, where $\mathcal{F}_{e q}$ is given by (1) with $r$ replaced by the appropriate $\bar{\rho}$. But in the shock region, $\rho_{a}<\rho_{b}$, this inequality is reversed: $\mathcal{F}(\{\rho(x)\}) \leq \mathcal{F}_{e q}(\{\rho(x)\})$ (as is clear from (5), since in region $B_{1}\left(A_{1}\right), y=0(y=1)$ gives $\left.\mathcal{F}_{\text {eq }}(\{\rho(x)\})\right)$. This is similar to what fluctuating hydrodynamics predicts for the Rayleigh-Bénard problem discussed earlier: fluctuations are decreased when $T_{a}>T_{b}$ (at least when $T_{a}-T_{b}$ is very small) and are increased when $T_{a}<T_{b}$, even in the stable conductance regime [12]. 
(d) In the symmetric case discussed in [11], as in an equilibrium system not at a phase transition (in any dimension), the probability of small fluctuations about the typical state can be obtained from $\mathcal{F}$ as a limit. More precisely if we write $\rho(x)=\bar{\rho}(x)+\frac{1}{\sqrt{L}} u(x)$ and then expand $\mathcal{F}$ to second order (the first order term being zero) we get a Gaussian distribution for $u(x)$ with covariance $C\left(x, x^{\prime}\right)$, where $C^{-1}\left(x, x^{\prime}\right)=\delta^{2} \mathcal{F} / \delta \rho(x) \delta \rho\left(x^{\prime}\right)$ evaluated at $\rho=\bar{\rho}$. This covariance is the suitably scaled microscopic truncated pair correlation [7]. For the asymmetric case discussed here the distribution of fluctuations need no longer be given by the LDF; in fact, $\delta^{2} \mathcal{F} / \delta \rho(x) \delta \rho\left(x^{\prime}\right)$ is discontinuous at $\bar{\rho}=1 / 2$ in the interior of region $C$ of the phase diagram, i.e., where $\rho_{a}>1 / 2>\rho_{b}$. Furthermore, the fluctuations there are no longer Gaussian.

To see this discontinuity in an explicit example let $\rho(x)=\frac{1}{2}+\epsilon \Theta(x-s)$, with $\rho_{b} \leq \frac{1}{2}-\epsilon \leq \rho_{a}$; here it is easy to compute $\tilde{\mathcal{F}}(\epsilon) \equiv \mathcal{F}(\{\rho(x)\})$. First, $F_{\rho}(x)=1-\rho(x)$ if $\epsilon>0$ and $F_{\rho}(x)=\frac{1}{2}-\epsilon(1-s)$ if $\epsilon<0$; the constancy of $F_{\rho}$ for $\epsilon<0$ is due to the concave envelope construction (6). Then from (4) (we give only the small $-\epsilon$ behavior):

$$
\tilde{\mathcal{F}}(\epsilon) \simeq \begin{cases}4(1-s) \epsilon^{2}+\ldots, & \text { if } \epsilon>0, \\ 4(1-s)\left(1-\frac{s}{2}\right) \epsilon^{2}+\ldots, & \text { if } \epsilon<0 .\end{cases}
$$

The discontinuity of $\partial^{2} \tilde{\mathcal{F}}(\epsilon) / \partial \epsilon^{2}$ at $\epsilon=0$ signals that the fluctuations are anomalous (non-Gaussian). (Note that for $s=0, \partial^{2} \tilde{\mathcal{F}}(\epsilon) / \partial \epsilon^{2}$ is continuous at $\epsilon=0$ and is in fact equal to the inverse of the variance in the total number of particles [11].)

These non-Gaussian fluctuations can be observed by considering the total number $N_{s}$ of particles on lattice sites in $[s, 1]$, i.e., sites $i$ with $s L \leq i \leq L$. A calculation using the results of [13 for the microscopic probabilities shows that $\left[N_{s}-(1-s) L / 2\right] / \sqrt{L}$ converges, as $L \rightarrow \infty$, to a random variable $\mu$ with a well defined but nonsymmetric (and non-Gaussian) distribution having density

$$
p(\mu)=\frac{8}{\pi s^{3 / 2}(1-s)^{2}} \int_{0}^{\infty} d t t^{2} e^{-\left[\frac{t^{2}}{s}+\frac{2\left(2 \mu^{2}+2 \mu t+t^{2}\right)}{1-s}\right]} .
$$

This is in contrast with equilibrium systems (not at a phase transition) for which statistical mechanics [14 predicts Gaussian fluctuations of the number of particles in a macroscopic region (the variance being related to the compressibility). For large values of $|\mu|$, (8) yields

$$
-\log p(\mu) \simeq \begin{cases}\frac{4 \mu^{2}}{1-s}, & \text { if } \mu \gg 1, \\ \frac{4 \mu^{2}}{(1-s)(1+s)}, & \text { if } \mu \ll-1 .\end{cases}
$$

This agrees with the results of a large deviation calculation [11] of the probability $\exp [-L \hat{\mathcal{F}}(\epsilon)]$ of observing mean density $\frac{1}{2}+\epsilon$ in the interval $[s, 1]$, with no other constraints: the small $\epsilon$ behavior of $\hat{\mathcal{F}}(\epsilon)$ agrees with the large- $\mu$ asymptotics of (9) under the identification
$\mu=\sqrt{L}(1-s) \epsilon$. (Note that (9) differs from (7) because for (7) a constraint is imposed also in the region $[0, s]$.)

Derivation: To obtain $\mathcal{F}$ in (位) and (5) we use the exact expression for the measure $P_{L}(\tau)$ provided by the matrix method [5]. However, rather than calculating the probability of a given macroscopic profile $\rho(x)$ directly by summing $P_{L}(\tau)$ over all configurations $\tau$ corresponding to that profile, as we did for the symmetric case in [7], we follow here a different path, which has its origin in an $a$ posteriori observation made in [7. We noted there that while $\mathcal{F}_{s}\left(\{\rho(x)\} ; \rho_{a}, \rho_{b}\right)$ is nonlocal, it possesses a certain "additivity" property which, if it could have been established independently, would have yielded $\mathcal{F}_{s}$. This is exactly what we do for the ASEP: we first derive an additivity property from the matrix representation of $P_{L}(\tau)$, then use the additivity to obtain $\mathcal{F}$. Full details are given in 11]; here we give only a partial sketch of the arguments.

Let us introduce the $\operatorname{LDF} \mathcal{F}_{[a, b]}\left(\{\rho(x)\} ; \rho_{a}, \rho_{b}\right) \simeq$ $L^{-1} \log P_{L(b-a)}\left(\{\rho(x)\} ; \rho_{a}, \rho_{b}\right)$ for a system of $L(b-a)$ lattice sites in contact with reservoirs at densities $\rho_{a}$ and $\rho_{b}$. Let $K\left(\rho_{a}, \rho_{b}\right)$ be as in (3) and define

$$
\begin{aligned}
\mathcal{H}_{[a, b]}\left(\{\rho(x)\} ; \rho_{a}, \rho_{b}\right) & (10) \\
& \equiv \mathcal{F}_{[a, b]}\left(\{\rho(x)\} ; \rho_{a}, \rho_{b}\right)+(b-a) K\left(\rho_{a}, \rho_{b}\right) .
\end{aligned}
$$

It is shown in 11] that for $\rho_{a}>\rho_{b}$ we have

$$
\begin{array}{r}
\mathcal{H}_{[a, b]}\left(\{\rho(x)\} ; \rho_{a}, \rho_{b}\right)=\sup _{\rho_{b} \leq \rho_{c} \leq \rho_{a}}\left[\mathcal{H}_{[a, c]}\left(\{\rho(x)\} ; \rho_{a}, \rho_{c}\right)\right. \\
+ \\
\left.+\mathcal{H}_{[c, b]}\left(\{\rho(x)\} ; \rho_{c}, \rho_{b}\right)\right] .(11
\end{array}
$$

while for $\rho_{a}<\rho_{b}$,

$$
\begin{aligned}
& \mathcal{H}_{[a, b]}\left(\{\rho(x)\} ; \rho_{a}, \rho_{b}\right)(12) \\
&=\min \left[\mathcal{H}_{[a, c]}\left(\{\rho(x)\} ; \rho_{a}, \rho_{a}\right)+\mathcal{H}_{[c, b]}\left(\{\rho(x)\} ; \rho_{a}, \rho_{b}\right),\right. \\
&\left.\mathcal{H}_{[a, c]}\left(\{\rho(x)\} ; \rho_{a}, \rho_{b}\right)+\mathcal{H}_{[c, b]}\left(\{\rho(x)\} ; \rho_{b}, \rho_{b}\right)\right] .
\end{aligned}
$$

Equations (11) and (12) are the additivity relations for the ASEP.

In this letter we will sketch a derivation of (11) in the special case $q=0$; for the general case and for the derivation of 122) see 11]. We use the matrix formula [5] for a system of $N$ sites:

$$
P_{N}(\tau)=\frac{\left\langle\rho_{a}\right| \Pi_{i=1}^{N}\left(D \tau_{i}+E\left(1-\tau_{i}\right)\left|\rho_{b}\right\rangle\right.}{\left\langle\rho_{a}\left|(D+E)^{N}\right| \rho_{b}\right\rangle},
$$

where the operators $D, E$ and vectors $\langle\rho|,| \rho\rangle$ satisfy

$$
\begin{aligned}
D E & =D+E, \\
\langle\rho| E=\frac{1}{\rho}\langle\rho|, & D|\rho\rangle=\frac{1}{1-\rho}|\rho\rangle .
\end{aligned}
$$

If (15) is extended to complex values of $\rho$ we may write the exact additivity formula

$$
\frac{\left\langle\rho_{a}\left|X_{0} X_{1}\right| \rho_{b}\right\rangle}{\left\langle\rho_{a} \mid \rho_{b}\right\rangle}
$$




$$
=\frac{1}{2 \pi i} \oint \frac{\left(\rho_{a}-\rho_{b}\right) d \rho}{\left(\rho_{a}-\rho\right)\left(\rho-\rho_{b}\right)} \frac{\left\langle\rho_{a}\left|X_{0}\right| \rho\right\rangle}{\left\langle\rho_{a} \mid \rho\right\rangle} \frac{\left\langle\rho\left|X_{1}\right| \rho_{b}\right\rangle}{\left\langle\rho \mid \rho_{b}\right\rangle},
$$

where $X_{0}, X_{1}$ are arbitrary polynomials in $D$ and $E$ and the contour is a circle $|\rho|=R$ with $\rho_{b}<R<\rho_{a}$. To obtain (16), note that it suffices to take $X_{i}=E^{p_{i}} D^{q_{i}}$, since from (14) any polynomial in $D$ and $E$ can be written as a sum of such terms. The cases $q_{0}=0$ or $p_{1}=0$ are immediately obtained from (15) and the residue calculus; the general case follows by an inductive argument, as the case $q_{0}, p_{1}$ can be reduced to the cases $q_{0}-1, p_{1}$ and $q_{0}, p_{1}-1$ using (14) on the left hand side of (16) and the corresponding identity $(1-\rho)^{-1} \rho^{-1}=(1-\rho)^{-1}+\rho^{-1}$ on the right hand side. Now the weights $\left\langle\rho_{a}\left|X_{0}\right| \rho\right\rangle /\left\langle\rho_{a} \mid \rho\right\rangle$ and $\left\langle\rho\left|X_{1}\right| \rho_{b}\right\rangle /\left\langle\rho \mid \rho_{b}\right\rangle$ in (16) are polynomials in $1 /(1-\rho)$ and $1 / \rho$, respectively, with positive coefficients, so that the integrand has a Laurent series for $\rho_{b}<|\rho|<\rho_{a}$ with positive coefficients and hence is a convex function of real $\rho$ for $\rho_{b}<\rho<\rho_{a}$; the minimum $\rho_{\text {min }}$ for such real $\rho$ (which must occur since there are poles at $\rho=\rho_{a}$ and $\left.\rho=\rho_{b}\right)$ is a saddle point for the complex integral. Thus, if $\rho(x)$ is a given profile and $X_{0}$ (respectively $X_{1}$ ) is a sum of products of $L(c-a)$ (respectively $L(b-c)$ ) factors of $D$ or $E$ consistent with the left (respectively right) part of this profile, and we assume that that the weights depend exponentially on $L$, we expect the integral to be dominated by this saddle point, leading to

$$
\begin{aligned}
& \frac{1}{L} \log \frac{\left\langle\rho_{a}\left|X_{0} X_{1}\right| \rho_{b}\right\rangle}{\left\langle\rho_{a} \mid \rho_{b}\right\rangle} \\
& \simeq \inf _{\rho_{b} \leq \rho \leq \rho_{a}}\left\{\frac{1}{L} \log \frac{\left\langle\rho_{a}\left|X_{0}\right| \rho\right\rangle}{\left\langle\rho_{a} \mid \rho\right\rangle}+\frac{1}{L} \log \frac{\left\langle\rho\left|X_{1}\right| \rho_{b}\right\rangle}{\left\langle\rho \mid \rho_{b}\right\rangle}\right\} .
\end{aligned}
$$

Using (17), (13), and the relation

$$
N^{-1} \log \frac{\left\langle\rho_{a}\left|(D+E)^{N}\right| \rho_{b}\right\rangle}{\left\langle\rho_{a} \mid \rho_{b}\right\rangle} \simeq-K\left(\rho_{a}, \rho_{b}\right),
$$

we obtain (11). Thus the rather surprising supremum in (11), which corresponds to a choice of the least probable alternative, arises mathematically through the contour representation (16) and its domination by a real saddle point; we still lack a physical explanation of (17).

To go from (11) to (41) we divide our system into $n$ parts of equal length, the $k^{\text {th }}$ interval being $[(k-1) / n, k / n]$, $k=1, \ldots, n$. Now note that for very large $n$ most of these intervals must have reservoir densities $\rho_{k-1}, \rho_{k}$ which are nearly equal, and that the LDF for these intervals is approximately given by (11) with $r \simeq \rho_{k-1} \simeq \rho_{k}$. On the other hand, the total length of the intervals for which this is not true (corresponding to points of discontinuity in the function $F_{\rho}$ ) will approach 0 as $n \rightarrow \infty$; these considerations lead then directly to (बi) in this limit. We pass from (12) to (5) by a similar process of subdivision, but the argument is even simpler since each interval except one has equal reservoir densities.

Conclusion: It would be interesting to know how kev results for the our simple model - the additivity (11212) for the LD functions, the suppression or enhancement of deviations of the density profile from its typical value as the reservoirs and the internal field cooperate or compete, and the non-Gaussian fluctuation (8) of the number of particles in a box of length $c L, 0<c<1$ - extend to more realistic systems, and whether they could be understood by a dynamical approach, as is done for the symmetric case in 15

We thank T. Bodineau, G. Giacomin, and J. M. Ortiz de Zárate for helpful discussions. The work of J. L. Lebowitz was supported by NSF Grant DMR-9813268, AFOSR Grant F49620/0154, DIMACS and its supporting agencies, and NATO Grant PST.CLG.976552. J.L.L. acknowledges the hospitality of the Institut Henri Poincaré, where a part of this work was done.

* Electronic address: derrida@lps.ens.fr

$\dagger$ Institute for Advanced Study, Princeton, NJ 08540 and Department of Physics, Rutgers; Electronic address: lebowitz@math.rutgers.edu

‡ Electronic address: speer@math.rutgers.edu

[1] D. Ruelle, Nature 441, 263 (2001)

[2] M. C. Cross and P. C. Hohenberg, Rev. Mod. Phys. 65, 851 (1993).

[3] S. Sasa and H. Tasaki, cond-mat/0108365 and references therein.

[4] J. Krug, Phys. Rev. Lett. 67, 1882 (1991)

[5] B. Derrida, M. R. Evans, V. Hakim and V. Pasquier, J. Phys. A 26, 1493 (1993); F. H. L. Essler, V. Rittenberg J. Phys. A 29, 3375 (1996); N. Rajewsky, M. Schreckenberg Physica A 245, 139 (1997); T. Sasamoto, J. Phys. A 32, 7109 (1999); R. A. Blythe, M. R. Evans, F. Colaiori, F. H. L. Essler, J. Phys. A 33, 2313 (2000).

[6] S. Olla, Probab. Th. Rel. Fields 77, 343 (1988); R. Ellis, Entropy, large deviations, and statistical mechanics (Springer, New York, 1985).

[7] B. Derrida, J. L. Lebowitz, E. R. Speer, Phys. Rev. Lett. 87, 150601(2001); J. Stat. Phys. 107, 599 (2002).

[8] H. Spohn, Large Scale Dynamics of Interacting Particles (Springer-Verlag, Berlin, 1991), and references therein.

[9] V. Popkov, G.M. Schütz, Europhys. Lett. 48, 257-263 (1999)

[10] T. M. Liggett, Stochastic interacting systems: contact, voter, and exclusion processes (Springer-Verlag, New York, 1999).

[11] B. Derrida, J. L. Lebowitz, and E. R. Speer, in preparation.

[12] P. N. Segrè, J. V. Sengers, Physica A 198, 46 (1993); P. N. Segrè, P. Schmitz, J. V. Sengers, Physica A 195, 31 (1993).

[13] K. Mallick, J. Phys. A 29, 5375-5386 (1996)

[14] H.-O. Georgi, Gibbs measures and phase transitions (W. de Gruyter, Berlin, 1988).

[15] L. Bertini, A. De Sole, D. Gabrielli, G. Jona-Lasinio, C. Landim, Phys. Rev. Lett. 87, 040601 (2001); condmat/0108040. 